\title{
TACTILE STEREOGNOSIS AND SYMBOLIA; HAVE THEY LOCALIZATION IN THE CEREBRAL CORTEX?*
}

\author{
By Morton Prince, M.D.,
}

OF BOSTON.

PROFESSOR OF NERVOUS DISEASES, TUFTS COLLEGE MEDICAL SCHOOL; PHYSICIAN FOR NERVOUS DISEASES, BOSTON CITY HOSPITAL,

Before discussing the localization of stereognosis, we ought to have an understanding as to what we are talking about.

The earlier writers on astereognosis (for instance, Hoffmann, Sailer, Dercum, Walton and Paul) commonly identified this defect with the failure of recognition of objects, and the practical test usually employed to determine its presence was to place objects in the hand of the subject and ask him to identify them. This conception is still largely and rather blindly, as it would seem, adopted by many recent writers (Mills and Wei. senburg, Campbell, Russell and Horsley, Starr, Cushing and others). Although stereognosis, strictly speaking, means the perception of solidity or form only, it might be insisted that the question of the use of the term is one of definition, and that there is no particular harm, beyond the fact of a bad terminology, in using stereognosis for tactile recognition of objects so long as it is merely a matter of description of a syndrome. When, however, it comes to a question of localization in the cerebral cortex of what these writers have been pleased to call the stereognostic "sense," at once a confusion of ideas as well as errors in our scheme of cerebral localization arise. Fron the very first, when astereognosis came to be studied as a syndrome, it was realized that the recognition of objects by tactile perception was a very complex affair and depended not only upon the correlation of a large number of subsidiary sense impressions, which included tactile, position, localization, movement, pressure, temperature, impressions, etc., but upon the perception of form and the interpretation of the whole

*Read at the thirty-third annual meeting of the American Neturological Association, May 7,8 and 9, 1907. 
through higher intellectual processes which again included visual and other memorial images of the objects, as well as memories of past experiences. In other words, it is an intellectual process; yet all this, while thoroughly appreciated, it should be said, Walton and Paul, for instance, proceeded to call a stereognostic sense and to localize in the Rolandic area.

Now, properly speaking, stereognostic perception is the perception of form, alone, in three dimensions and though this again is itself a complex conception, and not a sense ${ }_{2}$ it is far less complex than the notion of the nature of an object.

This distinction between stereognostic perception and the recognition of objects by touch is now clearly recognized by some recent and more precise writers (Raymond and Egger, Dejerine, Grasset, Claparde), and has led to some interesting observations on the defects of form-perception and object-perception and their interpretation (agnosia, asymbolia, tactile aphasia, etc.), which I shall presently refer to.

As to object-perception, aside from the theoretical unlikelihood of a complex intellectual process being localized in a small focal area within one or two convolutions, an improbability that is based in part on the absence of all analogy with anything that is known of intellectual processes, there is absolutely no clinico-pathological evidence that requires such a cortical localization. The localization of such an intellectual process is at best only an interpretation of the clinical findings and these, if carefully studied, will be found, I believe, to be insufficient for an interpretation of this kind. For as the intellectual notion of an object depends upon the integrity of the subsidiary sense perceptions, and as in every case where sensibility has been thoroughly tested in all its forms and where there has been loss or impairment of the notion of objects, there has been also more or less impairment of the subsidiary sense impressions, the loss of the notion can be equally well and more logically interpreted as due to insufficient information from the sensory loss. All that the clinico-pathological evidence justifies is the localization of the subsidiary sense impressions. The practical importance of clinically distinguishing between form-perception, or stereognosis proper, and object-perception is conclusively shown by two cases recently reported: one by Raymond and Egger ${ }^{1}$ and one by

${ }^{1}$ Un cas d'aphasie tactile; Rev. Neurol., April 30, I906. 
Dejerine, ${ }^{2}$ in both of which there was preserved the ability to recognize form of objects in three dimensions with entire inability to recognize the nature of the objects. From the fact that their patient was unable to name the test object placed in the. affected hand, Raymond and Egger interpreted the syndrome as one of "tactile aphasia," analogous to word-deafness; but Dejerine, who promptly brought forward a similar case, argued with convincing logic that the condition was not one of aphasia, as the question was not one of naming only, but of recognition; for the patient had no notion of the nature of the test object, and of course could not name it. As an interpretation, he pointed out that the failure to recognize the object could be explained by the impairment of certain subsidiary sense perceptions, for although the tactile and various other senses were preserved, yet there was marked impairment of the localizing perception (one of the most important), and some of the knowledge of the position of the fingers; and though what remained might well be sufficient for form-perception (stereognosis) it was not sufficient for object-recognition.

I would also point out in corroboration of this view (what seems to have escaped notice) that the answers of both subjects (Raymond and Egger's and Dejerine's) to questions regarding the form, though showing a perception of three dimensions, was far from sufficient to allow a notion of the object to be gained. For instance, who could recognize from the following answers, showing form, the nature of the test object:

OBJECT.

RIGHT (AFFECTED) HAND.

Thimble.

It is small. I have my finger in it. I do not know what it is.

Porcelain button, flat, It is a small object which has a bottom. It is with four holes.

A large key. a thimble.

It is of steel. It is longer than my hand. It is a handle. [At the moment when the thumb enters the ring] It is a pair of scissors.

Dessert spoon longer than the hand.

It is smooth. It is of metal. It is longer than my hand. It is a pair of scissors.

Orange.

It is large. It is round. It is rough. It is hard.

Pocket handkerchief. It is soft. It is wrinkled. It is not of cotton or paper.

${ }^{2}$ Considerations sur la soi-disant 'aphasie tactile'; Rev. Neurol., Ju1y I5, 1906 . 
Surely, even with the preservation of most of the tactual perceptions, unless the patient has more conception of form than that given by three dimensions, and has various tactual sensations, he can not recognize the nature of the object. Touch, too, must not only be quantitatively but qualitatively preserved. As Dejerine well says, therefore, Raymond and Egger's patient and his own were unable to name the objects from lack of information, not from tactile aphasia. The condition he terms agnosia.

Claparède, ${ }^{3}$ discussing the above papers, has suggested the following terminology: Astereognosis is equivalent to a trouble of what Wernicke has. called "prinuary identification"; what may be called simple perception or sensory recognition. It is, therefore, a trouble of the perception of the form of objects. The notion of an object is intellectual recognition. He proposes the term "Asymbolia" for a trouble of this process. Both troubles, that of primary identification and that of secondary identification, are forms of Agnosia. Agnosia may, therefore, be subdivided as follows:

Agnosia.-(a) Trouble of the primary identification; primary agnosia. (b) Trouble of the secondary identification: asymbolia.

As these terms are also applicable to recognition through other senses, it logically follows that in the domain of touch the primary identification would be called "tactile astereognosis"; while the trouble of secondary identification would be called "tactile asymbolia." There would naturally be numerous transitional forms.

Claparède, however, goes farther and holds that inasmuch as asymbolia is an intellectual defect, and in his view due to a defect in the cerebral association connections, that it may exist without loss of either stereognosis or sensory troubles. This interpretation, however, he admits is theoretical and not based on actually observed data.

The intellectual element in perception of form and recognition of objects is brought out in testing those faculties in the use of the toes. Walton and Paul called attention to the inability of normal people to recognize form and objects with the feet, but their tests were insufficient, as they consisted simply in

\footnotetext{
${ }^{3}$ Agnosie et asymbolie; Rev. Neurol., Sept. 15, 1906.
} 
pressing an object against the sole of the foot. The limited information furnished by such a method would be insufficient even in the hand, as may be easily shown. The only proper test is to allow a person to palpate, i. e., grasp the object with the toes. Tests made in this way with the patient whose case is reported by me in the present number of The JournaL ${ }^{4}$ gave some instructive results. The case was one of hemiplegia, the leg being slightly affected. In the right (affected) foot there was no loss of tactile sense, as tested with the camel's hair brush. The localizing perception similarly tested showed only few mistakes on the plantar surface (more on the dorsal). The perception of movement of the toes of the affected foot was moderately impaired both for movement itself and direction, the big toe, which was unable to recognize large circles of circumduction, being most affected. The same was true for posture.

Now when the perception of form was tested by allowing him to palpate the objects with the toes and roll them about against the floor, it was found that form was about as well recognized with the right foot as with the normal left, as shown by his answers, although subjectively it was apparently better recognized on the left, the patient asserting that this appeared to him to be the case. The difference was not remarkable. In correctness the answers varied considerably as respects both feet, but apparently perception depended upon the nicety with which the object could be grasped. Once the perception on the right was substantially correct when a flat circular tin ointment box about one and one-half inch in diameter and onefourth inch deep was described as "round (i. e., circular) and flat on top, one and one-half to one and three-quarters inches in diameter"; but on repeating the test it was described as "not round," as was the case on both occasions on the left.

A small bottle.-Right, "round like a ball." Left, "like a penknife as I roll it over." Repeating test on right, he gave the same answer as on the left, but thought he palpated the bottle differently from the first time.

Square box.-Right, "round like a ball." Left, same.

Piece of erasing rubber.-Right, "long, something like your finger" (correct) ; "bevelled edges" (wrong). Left, same.

Small glass tubular bottle.-Right, "like a pencil." Left,

\footnotetext{
${ }^{4}$ A Study in Tactile Localization, etc.
} 
"larger" (correct). (Thought it elliptical in diameter owing to a ridge made by paper label becoming creased when rolled under foot).

The patient found it difficult to give a more extended description than that contained in the above answers. But as to the recognition of the object, whether by the normal left or by the right foot the patient had not in a single instance the remotest idea of what it was. A little consideration will make clear that this is what might have been anticipated, for however near his recognition approached to a true perception of form in three dimensions there was plainly insufficient information for recognition of the object itself, for he could not follow the details of form nor make more than a guess at the material. The fact that the failure of object recognition was due to failure of sufficient information to form an intellectual judgment, and not to the absence of a cortical center for tactile symbolia, is supported by the following interesting detail: When the subject was shown the test-objects (seven in all) before the tests were made, he was able to name the object in nearly every instance, making only two mistakes with the right foot and one with the left: viz., on the right a ball was called a "short bottle," and on both sides the necked-bottle was called the tubular bottle, the patient being unable to detect the change of form at the neck and therefore recognized only the tubular form.

When asked to analyze the subjective perception and describe how he identified the objects after having become visually familiar with them, he said there came into his mind a visual image of the object which he could "see under his foot." In other words, it would appear that, now knowing what sensations ought to go with each object, out of such sense impressions as were furnished he could construct the object subjectively and identify it. This is supported by the fact that he continued to see the necked-bottle as a tube-bottle even after knowing its true nature, for, as he said, the feeling was that of a tube without a neck, and this raised the image of that kind of a bottle.

By contrast [with the hand where there was profound alteration of various forms of sensibility (localization, movement. pressure)] he remained still unable to recognize objects after he had been made familiar with them by sight. That is to say, 
the sensory impressions from the hand were not sufficiently precise to allow him to identify them with the memorial images of those previously experienced. This interpretation is supported by the following observation: After considerable improvement had taken place in the sensibility of the palmar surface of the hand it was found that the perception of localization, when tested by Volkmann's method, was practically normal. This method, called also the "looking" procedure, consists in allowing the patient to open his eyes after a spot on the skin has been touched and directing him to point out the spot touched. He thus has the actual visual image of the hand to guide him in finding the spot. Now, when a screen was placed so that he could not see his hand and he was directed to mention which finger and which segment of the finger were touched, he was all at sea in his answers. The errors in localization were very wide and consisted in guessing the wrong finger as well as the wrong segment.

The explanation which the subject gave of the difference in the results of the two procedures I employed was as follows: By the "looking" method the visual image of his hand enabled him to recall the normal sensations belonging to each segment, and by comparing these memorial images with the sensation awakened by the test he could locate the latter. When, however, he was unable to see his hand, then he had no memorial images with which to judge and compare the test sensations which in themselves must have lacked the quality of "thereness" necessary to enable him to locate them without comparison. It is of course under the latter conditions (without vision) that stereognosis must necessarily be tested, and the observation shows how the perception of localization varies according to the method by which it is tested. This has been well brought out by Spearman ${ }^{5}$ in his excellent analysis of the various procedures.

Mills and Weisenburg" in a recent paper on "The Subdivision of the Representation of Cutaneous and Muscular Sensibility and of Stereognosis," meaning symbolia in the cerebral cortex, have maintained that the stereognostic zone is independent of the cutaneous and muscular sensibility and also of the motor area, and, following Mills' previously expressed views,

${ }^{5}$ Analysis of localization, etc. Br. Journal of Psychology, January, 1905.

${ }^{6}$ The Journal of Nervous and Mental Disease, October, Igo6. 
that the streognostic (i. e., symbolic) area is located in the posterior parietal region. (What they mean by stereognosis is not perfectly clear, as they do not definitely distinguish between stereognosis and the intellectual recognition of the object; but their language and the tests employed identify the two and make astereognosis the equivalent of asymbolia.)

They report three cases, in two of which their so-called astereognosis was confined to the three outer fingers of the affected hand. In the third case, while there was general asymbolia of the whole hand, they note what would appear to be a significant fact, to be later referred to, that there was loss in these same fingers of the perception of angularity of objects. From these findings they draw the conclusion that there is a subdivision of the stereognostic perception in a definite cortical area of the brain which they localize as above mentioned. A careful study of these cases, however, shows that they prove too much for this interpretation.

In the first case there was at one time impairment or loss of the important tactual perceptions (including that of position) in the whole affected hand and with this defect intellectual recognition of objects (called by the authors "stereognostic conception") was also lost in the whole hand. Later the loss of sensibility largely cleared up in the thumb and forefinger, where the "sense of position" became normal and with this clearing up the patient regained the ability to recognize objects between these digits, but could not do so with the other three fingers where sensibility remained impaired. Later, when loss of sensibility again extended as before over the whole hand, he was again unable to recognize objects over the whole hand. With partially lost sensibility there was partial asymbolia; with completely lost sensibility, complete asymbolia.

In the second case Spiller had found loss of "stereognostic perception" apparently in the whole affected hand, corresponding to loss of perception of position, localization and movement (Spiller does explain what is to be understood by stereognostic perception). Later Weisenburg found that the losi of sensibility had largely cleared $u p$ in the thumb and forefinger, but persisted in the three ulnar fingers and correspond. ingly, be it noted, that he could recognize objects with the thumb and forefinger, but not with the others. 
It seems to me these findings are pregnant with meaning, not as showing the subdivision of the "stereognostic conception" center in the cortex, but as showing that with the subdivision of information given by the tactual senses there is a subdivision of the intellectual ability to recognize objects. If the "stereognostic conception" had a special center of its own and particularly if, as the authors believe, separated from the tactual centers, we should not expect this recession and expansion of the "stereognostic conception" pari passu with recession and expansion of the tactual perceptions. We should expect that they would not be absolutely co-extensive as they are, but that stereognostic loss from lesion of one center would at times ex. ceed, or be present without loss of, sensibility due to lesion of another center. Tactual defects always occur with tactile asymbolia.

Stereognosis is plainly an intellectual process and to attempt to localize it is to do that for which there is no analogy in the scheme of cerebral localization. Intellectual judgments are not thus far, according to data at hand, localizable nor likely to be. The nearest approach to such a scheme is that of Marie's new theory of aphasia, according to which aphasia is an intellectual defect and localized in Wernicke's zone. This, to my way of thinking, is the weak point in Marie's theory. It is true that Marie defines the intellectual defect as a special intellectual function, but he fails to define what he means by a "special function," though holding that it is not one of special sensory images-visual, auditory, etc.

Though Marie may be quite right in his general theory, and in the localization of his intellectual aphasia in Wernicke's zone, his denial that the special intellectual defect consists of a loss of sensorial images may be wrong, and does not seem to be warranted by any evidence that he brings forward; and it may well be, therefore, that if his localization is correct, it is only a localization of sensorial images.

This denial of sensorial images in the mechanism of aphasia seens to weaken his theory rather than to strengthen it. Certainly, the localization of broad intellectual processes does not at present rest upon any sound basis of fact.

The only conclusive evidence for the localization of symbolia must rest upon clinical observations in which there is found to 
be loss of power to recognize objects by touch, without loss of the subsidiary sensations and perceptions, and the final demonstration of focal localization in the cortex. No such case, at least no case exhaustively examined, so far as I have been able to find, with or without anatomical findings, has thus far been reported. In the absence of such a case there is absolutely no logical warrant in attempting to treat symbolia as a sense or concept or function and find for it a localization. Symbolia can only be spoken of and treated as a function symbolically, as a convenient expression to represent a complex process, and although it may be legitimate to do this for purposes of convenience, we are at once led into error when we attempt to localize the symbol. In localizing symbolia, we are interpreting the evidence which in every case involves the impairment of different forms of sensation; and the interpretation which localizes the tactual impressions and makes the symbolia depend upon the loss of sufficient information for judgment fulfills all the logical requirements of the case.

If the clinico-pathological evidence can not be interpreted as indicating the localization of asymbolia, the infellectual recognition of an object, can it be interpreted as indicating a localization of true stereognosis or form perception?

The tri-dimensional recognition of form is a complex process, though not so complex as that of identification of an object. It is dependent upon the integrity of the subsidiary sensations and perceptions. Though tri-dimensional perception, that is, of solidity (stereognosis), is not of itself sufficient for secondary (inteltellectual) idcntification, it must shade through intermediate forms into the latter as the perception of curves, angles and shape become added. Yet it is theoretically possible that in the simpler and in many of these intermediate forms, the subsidiary sensations and perceptions may become so organized as to permit of its definite localization as a quasi-function in a so-called cortical stereognostic center. A definite proof of this interpretation must, however, as with symbolia, depend upon the clinical demonstration of loss of the stereognostic sense coincident with integrity of the subsidiary tactual sensations and perceptionstouch, localization, movement, etc. In the absence of such demonstration and in the presence of impairment of one or more of the latter, we plainly are not justified in interpreting astereognosis 
proper as a loss of a special function with a special center, but only as due to impairment of the subsidiary perceptions upon which our recognition of form depends; that is, to loss of sufficient information.

The above analysis and criticism of Mills and Weisenburg's two cases is also applicable to them in respect to the perception of form (stereognosis proper) which I think we may safely assume was lost in these cases because of the profound impairment of localization, etc. The third case bears distinctly on this point. Here was inability "to recognize an object placed in any part of the hand." Nevertheless, the patient could recognize "angularity of all objects with the thumb and forefinger," but not with the other fingers, and corresponding to this difference, sensibility was least impaired in the thumb and forefinger. Perception of angularity is of form, and this varied with the condition of sensibility. The sensory information from the forefinger and thumb, as in Raymond and Egger's and Dejerine's cases, was sufficient for the recognition of form but not for recognition of objects.

Whether or not then, stereognosis and symbolia are to be regarded as having a definite cortical localization depends primarily upon the interpretation of the clinical evidence. The localization of the subsidiary sensations and perceptions is all that this evidence warrants. Theoretically, it is not impossible that the association of the sensory perceptions involved in the recognition of form may become so highly organized that the correlated process may be localized in a definite center, but this is far from proved.

The terms "tactile asymbolia" and "tactile astereognosis" ought, I believe, either to be dropped from our symptomatology, or retained as symbols of complex tactual defects. We do not coin terins to define faulty judgments in other sensory fields; when vision is impaired so that objects are not recognized, we do not say there is visual asymbolia, and so with the other senses. If the terms are retained, they should be used only as convenient age careful examination of the various forms of tactual defects The disadvantage of such terms is that their use tends to discourage the careful examination of the various forms of tactual defects in individual cases and to confusion in cerebral localization. 